\title{
Robot-assisted right colectomy: surgical technique and review of the literature
}

\author{
Wojciech Witkiewicz ${ }^{1,2}$, Marek Zawadzki ${ }^{1}$, Marek Rząca ${ }^{1}$, Zbigniew Obuszko' ${ }^{1}$, Roman Czarnecki ${ }^{1}$, Jakub Turek ${ }^{1}$, \\ Stawomir Marecik ${ }^{3,4}$ \\ ${ }^{1}$ Regional Specialized Hospital, Research and Development Center, Wroclaw, Poland \\ 2Faculty of Medicine, Wroclaw Medical University, Poland \\ 3Department of Colon and Rectal Surgery, Advocate Lutheran General Hospital, Park Ridge, IL, USA \\ ${ }^{4}$ Department of Colon and Rectal Surgery, University of Illinois at Chicago, Chicago, IL, USA
}

Videosurgery Miniinv 2013; 8 (3): 253-257

DOI: $10.5114 /$ wiitm.2011.33761

\begin{abstract}
Following the successful introduction of robotic surgery to the field of urology and gynecology, its use gained even more interest among those in the field of colorectal surgery. Rectal resection is believed to be among the best suited for robotic assistance. In particular, the right hemicolectomy procedure has been proposed as a training tool in order to gain clinical experience with the robot. This article and attached video demonstrates, in detail, the robot-assisted right hemicolectomy, including key landmarks of the procedure. The case presented involved a 58-year-old man with an advanced cecal adenocarcinoma. In our opinionrobot-assisted right colon resection is a procedure that offers particular value for the novice robotic team who is in the beginning stages of their colorectal surgery experience. Although no concrete advantages for use of the robot in this particular procedure have been demonstrated in the literature, because it is a relatively straightforward and simple procedure, it can serve as a valuable training tool for the novice robotic surgeon.
\end{abstract}

Key words: da Vinci robot, colon resection, colon cancer, robotic surgery.

\section{Introduction}

Laparoscopic procedures in general surgery have been performed successfully for more than 20 years. The first laparoscopic cholecystectomy was performed in 1985 by Mühe in Germany [1]. Since that time, substantial improvements in optic systems and instrumentation have made laparoscopic surgery much more accurate, safer, and easier to learn.

Technical advances have also allowed for more complicated procedures, such as colorectal resections, to be performed safely by laparoscopy [2]. Such procedures have become standard in numerous centers worldwide and many studies have demonstrated that laparoscopic colon resections are associated with lower perioperative morbidity, shorter hospital stay, and quicker recovery than with open colon resections [3-5]. The studies also showed that oncological outcomes following laparoscopic procedures are no worse than those following open resection $[6,7]$.

In light of these advances, and favorable results seen, it seems clear that laparoscopic colectomy should be considered the gold standard procedure for benign and malignant indications. Still, the percentage of laparoscopic colectomies performed is disap- 
pointingly low when compared with laparotomy, and this is true not only in Poland but also in the United States [8]. The main explanation offered for this is that it requires a longer learning curve and a need for extensive training of surgeons [9]. Another likely reason, however, is the fact that there is limited exposure to laparoscopic colorectal surgery in many of the hospitals in which surgeons are educated and trained.

In order to overcome these shortcomings and allow for more patients to benefit from a minimally invasive procedure, robotic surgery is often suggested as a potential solution. A favorable learning curve is frequently identified as one of the advantages of robotic surgery [10]. In fact, following the successful introduction of robotic surgery in the field of urology, cardiac and gynecologic surgery [11-13], its use gained more and more interest among those in the field of colorectal surgery [14-17].

From a technical standpoint, rectal resection is believed to be among the best suited for robotic assistance. Still, rectal resection for cancer is a complex and challenging procedure, particularly for the novice robotic surgeon. Because of this, the right hemicolectomy procedure has been proposed as a training tool in order to gain clinical experience with the robot [18].

In this article, we present technical details and discuss potential advantages of robot-assisted right hemicolectomy.

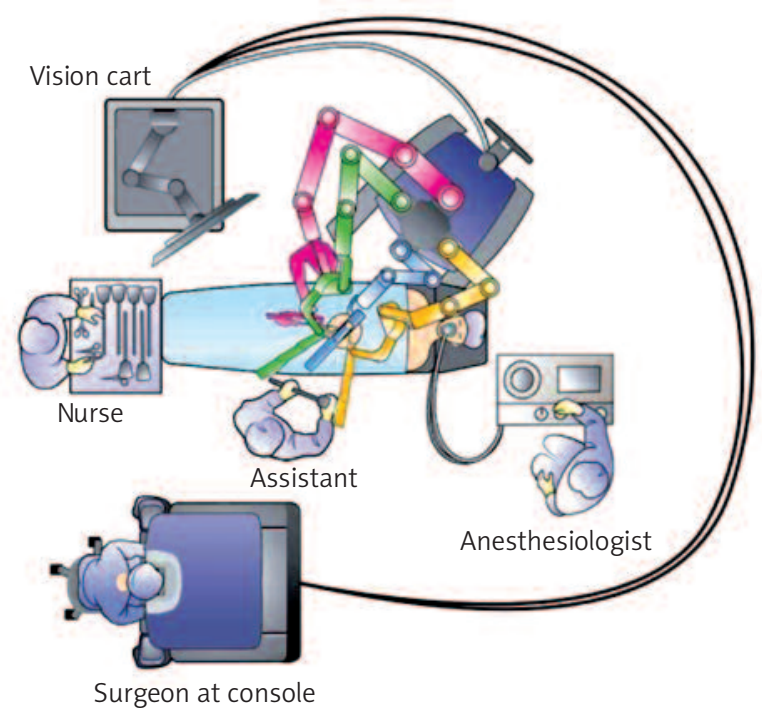

Figure 1. Patient and robot positioning

\section{Case report}

The da Vinci robot was obtained at the end of 2010 as the first robotic console in Poland. Between December 2010 and May 2012, our surgical team performed 80 robotic procedures, including 20 colorectal resections. Of these, there were 15 rectal resections and 5 right hemicolectomies.

The case presented herein involved a 58-year-old man with a body mass index (BMI) of $26 \mathrm{~kg} / \mathrm{m}^{2}$ and an advanced cecal adenocarcinoma. The attached video demonstrates, in detail, the robot-assisted right hemicolectomy, including key landmarks of the lateral to medial and inferior to superior approach. The procedure took $210 \mathrm{~min}$ and the estimated blood loss was $50 \mathrm{ml}$. The patient was discharged home on postoperative day 6 . The pathology report revealed a T4 adenocarcinoma with adequate lymph node harvest $(n=15)$.

\section{Surgical technique}

\section{Patient and robot positioning}

The da Vinci Surgical System consists of the robotic cart, the vision cart, and the surgeon's console (Figure 1). The entire system takes up a significant amount of space and ideally should be placed in a dedicated operating room of sufficient size.

For right hemicolectomy, the patient is placed in a supine or lithotomy position. The patient is then secured to the operating table with the help of a bean bag, with both arms tucked at bedside. Additional shoulder harnesses are placed in order to support the patient when in the Trendelenburg position. The robot is brought in from the right side and the bedside assistant and the scrub nurse are situated to the patient's left side. Once the robot is docked, there can be no change to the patient's position or the robot's position, without first undocking the robotic arms.

\section{Port placement}

As shown in Figure 2, port placement for the robotic procedure closely resembles the port configuration for laparoscopic right hemicolectomy. We routinely use only two of the three robotic working arms, along with a camera, although all three robotic working arms can be used if desired. One assistant laparoscopic port is added for additional retraction, as well as an energy device or an endostapler. 


\section{Procedure}

The procedure begins with diagnostic laparoscopy. The abdomen is inspected to determine the feasibility of minimally invasive resection and to identify the extent of disease. The patient is placed in the Trendelenburg position with the right side up. This allows for the small bowel and omentum to be displaced to the left upper quadrant, exposing the cecum and terminal ileum. The robot is then brought from the right side of the patient and docked onto the ports. We routinely use a robotic hook cautery on the left robotic arm and a bipolar fenestrated grasper on the right robotic arm. Depending on the surgeon's preference and anatomical variations, either a medial to lateral or lateral to medial approach can be used. In this case presented, a lateral to medial technique was applied. The cecum is grasped and retracted medially and the peritoneum incised in the right pericolic gutter. This step helps to open up the avascular retroperitoneal plane of dissection. In this plane, the entire right colon is mobilized up to the hepatic flexure. During this part of the dissection, the right gonadal vessels and the right ureter should be identified and preserved. Next, the ileocolic pedicle is controlled. At this point, the cecum is retracted laterally and the ileocolic artery is carefully dissected close to its origin. The artery is then transected using a suitable energy device. Alternative methods include vascular endostapling or suture-ligation with the robotic system.

The mobilization of the hepatic flexure is the next step. The table is tilted to the reverse Trendelenburg position, which allows for the omentum and the transverse colon to shift caudad, thus exposing the hepatocolic ligament. At this point, it is necessary to undock the robotic arms temporarily from the ports before changing position. The transverse colon is retracted inferiorly and the gastrocolic ligament divided with the help of bipolar coagulation or an energy device. The dissection is continued toward the hepatic flexure and the final attachments of the colon to the retroperitoneum are divided. This completes the mobilization of the entire right colon and the robotic part of the procedure.

Once complete, the robot is undocked and the incision for a camera port is extended superiorly to create a small midline minilaparotomy. The mobilized right colon is then exteriorized through this incision and resected. The standard side to side ileocolic anastomosis is created in open fashion.

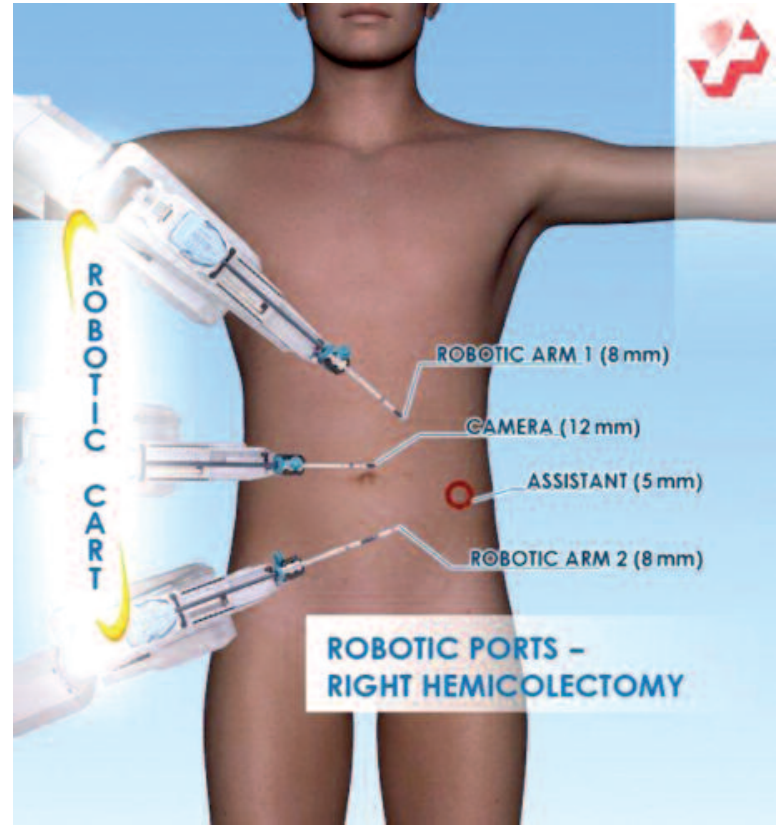

Figure 2. Port placement

\section{Discussion}

Since 2001, when Weber et al. [19] performed the first robot-assisted colon resection using the da Vinci Surgical System, the role of robotic colorectal surgery has been extensively studied in the literature. Over the past several years, a variety of studies have demonstrated the feasibility and safety of robotic colon and rectal resection. Still, while the benefit of robotic assistance in rectal tumors has been appreciated by a number of surgeons, no randomized trial has been published on this topic to date [15-17].

Advocates of the robot-assisted technique point to superior retraction, visualization, and dissection offered with the robot, resulting in a better mesorectal grade [20], earlier recovery of urogenital function [21], and lower conversion rates [15]. However, the benefit of robotic assistance in right hemicolectomy is less evident. Authors comparing robotic and laparoscopic right colectomy have demonstrated that robotic procedures are longer and more expensive $[18,22,23]$. Additionally, none were able to clearly document the advantage of the robot over traditional laparoscopy, as it relates to oncological outcomes.

DeSouza et al. retrospectively compared 40 robotassisted and 135 laparoscopic-assisted right hemicolectomies [18]. They showed the robotic procedure to be 40 min longer and more costly than its laparo- 
scopic counterpart. Additionally, there was no significant difference in surgical clearance, morbidity or length of hospital stay. The authors concluded that robotic right hemicolectomy could serve as a training tool for the novice robotic surgeon. The conclusion of these authors correlates well with our own initial observations.

From December 2010 to May 2012, our surgical team performed 20 robot-assisted colorectal resections. The case presented in this video article is the authors' second independent right colon resection. Despite the patient's advanced (T4) cecal lesion, the surgical team was able to achieve a clear plane of dissection with minimal blood loss and satisfactory tumor and lymph node clearance $(n=15)$.

With every new robotic technique comes its own learning curve. Right hemicolectomy has been found to be among the easiest of robotic colon resections. It can be safely performed with two robotic arms, one assistant instrument, and a camera. By employing only two of the three robotic arms, the novice robotic surgeon avoids the need to change between arms, which can prove difficult during the initial robotic experience. The lack of tactile sensation and the tissue damage in the mechanism of traction injury was also described as a robot-specific intraoperative complication [10]. Right colectomy again serves as a good in vivo training procedure because it allows for appropriate tissue grasping and retraction prior to attempting a more complex procedure such as rectal dissection.

As with laparoscopy, either the lateral to medial or the medial to lateral approach can be used for robotic right hemicolectomy and both approaches have been determined to be safe and feasible [18, 24]. In the current phase of our learning curve, the authors prefer the lateral to medial approach exclusively, as it most closely resembles the open technique. Robotic mobilization of the colon and separation of the ileocolic pedicle are performed as in the open approach, and the bowel division and creation of anastomosis are performed extracorporeally. This helps to make the technique safe, simple and effective.

The totally robotic right hemicolectomy has also been described in the literature. D'Annibale et al. reported 50 consecutive robotic right colectomies [25]. Of these, 45 required intracorporeal hand-sewn anastomosis. No major complications were noted in this group. The specimen was routinely retrieved through a Pfannenstiel incision. The authors concluded that the robotic system was highly suitable for performing microsutures and anastomoses. The authors also suggested that performing intracorporeal anastomosis enabled them to choose the optimal abdominal location for harvesting the specimen (Pfannenstiel incision). In contrast, other studies have shown no additional patient benefits from a totally intracorporeal approach [26, 27]. Moreover, the extracorporeal anastomosis is believed to be quicker and easier than attempting intracorporeal resection and anastomosis [28].

Ligation of the ileocolic pedicle can be performed intracorporeally or extracorporeally following exteriorization of the bowel. In particular, the intraabdominal ligation is recommended in the obese patient when significant mesenteric fat and a thick abdominal wall preclude exposure of the ileocolic pedicle [28]. We routinely control the ileocolic pedicle robotically, regardless of the BMI of the patient. In our opinion, endowristed robotic instruments greatly facilitate dissection of the vascular pedicle, making this step of the procedure relatively simple.

High cost remains a major limitation of robotic technology. Published reports consistently show a higher cost for robotic colectomy when compared to the cost of the laparoscopic procedure [18, 22, 23]. DeSouza et al. found robotic right hemicolectomy to be $20 \%$ more expensive than its laparoscopic counterpart [18]. The cost analyses included the initial cost of the robot, yearly maintenance, and instrumentation. The initial cost for the equipment has been found to account for most of the overall high cost of robotic surgery. In fact, the robotic platform alone costs $\$ 1.75$ million. Additional expenditures involve annual maintenance, which is approximately $\$ 150,000$, and the cost of disposable robotic instrumentation (each instrument can be used a maximum of 10 times). Of course, one primary reason for this high cost can be attributed to the fact that the robot is currently manufactured and distributed by a single company. We can only hope that new developments in the field of robotic surgery will create more competition, ideally resulting in lower cost for this robotic technology.

In conclusion, robot-assisted right colon resection is a procedure that offers particular value for the novice robotic team at the beginning stages of their colorectal surgery experience. Although no concrete advantages for use of the robot in this particular procedure have been demonstrated in the literature, because it is a relatively straightforward and simple 
procedure, it can serve as a valuable training tool for the novice surgeon. The entire surgical team, including the operator, assistant and scrub nurses, can acquire basic robotic skills necessary to safely progress to more complex procedures.

\section{Acknowledgments}

This publication is part of the project "Wrovasc Integrated Cardiovascular Centre", co-financed by the European Regional Development Fund, within Innovative Economy Operational Program, 2007-2013, carried out in the Regional Specialist Hospital, Research and Development Centre in Wroclaw.

\section{References}

1. Litynski GS. Erich Mühe and the rejection of laparoscopic cholecystectomy (1985): a surgeon ahead of his time. JSLS 1998; 2: 341-6.

2. Duda M, Gryga A, Czudek S, Skalický P. Twenty years of minimally invasive surgery in the Czech Republic. Videosurgery Miniinv 2011; 6: 42-7.

3. Lacy AM, Garcia-Valdecasas JC, Delgado S, et al. Laparoscopyassisted colectomy versus open colectomy for treatment of nonmetastatic colon cancer: a randomised trial. Lancet 2002; 359: 2224-9.

4. Nelson H, Sargent DJ, Wieand HS, et al.; the Clinical Outcomes of Surgical Therapy (COST) Study Group. A comparison of laparoscopically assisted and open colectomy for colon cancer. N Engl J Med 2004; 350: 2050-9.

5. Guillou PJ, Quirke P, Thorpe H, et al. Short-term endpoints of conventional versus laparoscopic-assisted surgery in patients with colorectal cancer (MRC CLASICC trial): multicentre, randomised controlled trial. Lancet 2005; 365: 1718-26.

6. Jayne DG, Guillou PJ, Thorpe H, et al. UK MRC CLASICC Trial Group Randomized trial of laparoscopic-assisted resection of colorectal carcinoma: 3-year results of the UK MRC CLASICC Trial Group. J Clin Oncol 2007; 25: 3061-8.

7. Skrovina M, Duda M, Srovnal J, et al. Evaluation of laparoscopic resection of colorectal carcinoma from the viewpoint of molecular biology. Videosurgery Miniinv 2012; 7: 19-26.

8. Delaney CP, Chang E, Senagore AJ, Broder M. Clinical outcomes and resource utilization associated with laparoscopic and open colectomy using a large national database. Annals Surg 2008; 247: 819-24.

9. Tekkis PP, Senagore AJ, Delaney CP, Fazio VW. Evaluation of the learning curve in laparoscopic colorectal surgery: comparison of right-sided and left-sided resections. Ann Surg 2005; 242: 83-91.

10. Bokhari MB, Patel CB, Ramos-Valadez DI, et al. Learning curve for robotic-assisted laparoscopic colorectal surgery. Surg Endosc 2011; 25: 855-60.

11. Ficarra V, Cavalleri S, Novara G, et al. Evidence from robot-assisted laparoscopic radical prostatectomy: a systematic review. Eur Urol 2007; 51: 45-55.
12. Gao C, Yang M, Wu Y, et al. Hybrid coronary revascularization by endoscopic robotic coronary artery bypass grafting on beating heart and stent placement. Ann Thorac Surg 2009; 87: 737-41.

13. Swan K, Advincula AP. Role of robotic surgery in urogynecologic surgery and radical hysterectomy: how far can we go? Curr Opin Urol 2011; 21: 78-83.

14. Zimmern A, Prasad L, Desouza A, et al. Robotic colon and rectal surgery: a series of 131 cases. World J Surg 2010; 34: 1954-8.

15. DeSouza AL, Prasad LM, Marecik SJ, et al. Total mesorectal excision for rectal cancer: the potential advantage of robotic assistance. Dis Colon Rectum 2010; 53: 1611-7.

16. Baik SH, Kwon HY, Kim JS, et al. Robotic versus laparoscopic low anterior resection of rectal cancer: Short term outcome of a prospective comparative study. Ann Surg Oncol 2009; 16: 1480-7.

17. Pigazzi A, Luca F, Patriti A, et al. Multicentric study on robotic tumor-specific mesorectal excision for the treatment of rectal cancer. Ann Surg Oncol 2010; 17: 1614-20.

18. deSouza AL, Prasad LM, Park JJ, et al. Robotic assistance in right hemicolectomy: is there a role? Dis Colon Rectum 2010; 53: 1000-6.

19. Weber P, Merola S, Wasielewski A, Ballantyne G. Teleroboticassisted laparoscopic right and sigmoid colectomies for benign disease. Dis Colon Rectum 2002; 45: 1689-96.

20. Baik SH, Kwon HY, Kim SJ, et al. Robotic versus laparoscopic low anterior resection of rectal cancer: short-term outcome of a prospective comparative study. Ann Surg Oncol 2009; 16: 1480-7.

21. Kim JY, Kim NK, Lee KY, et al. A comparative study of voiding and sexual function after total mesorectal excision with autonomic nerve preservation for rectal cancer: laparoscopic versus robotic surgery. Ann Surg Oncol 2012; 19: 2485-93.

22. Delaney CP, Lynch AG, Senagore AJ, Fazio VW. Comparison of robotically performed and traditional laparoscopic colorectal surgery. Dis Colon Rectum 2003; 46: 1633-9.

23. Rawlings AL, Woodland JH, Vegunta RK, Crawford DL. Robotic versus laparoscopic colectomy. Surg Endosc 2007; 21: 1701-8.

24. Ballantyne GH, Ewing D, Pigazzi A, Wasielewski A. Telerobotic assisted laparoscopic right hemicolectomy: lateral to medial or medial to lateral dissection? Surg Laparosc Endosc Percutan Tech 2006; 16: 406-10.

25. D'Annibale A, Pernazza G, Morpurgo E, et al. Robotic right colon resection: evaluation of first 50 consecutive cases for malignant disease. Ann Surg Oncol 2010; 17: 2856-62.

26. Bernstein MA, Dawson JW, Reissman P, et al. Is complete laparoscopic colectomy superior to laparoscopic assisted colectomy? Am Surg 1996; 62: 507-11.

27. Roscio F, Bertoglio C, De Luca A, et al. Totally laparoscopic versus laparoscopic assisted right colectomy for cancer. Int I Surg 2012; 10: 290-5.

28. Young-Fadok TM. Nelson H. Laparoscopic right colectomy fivestep procedure. Dis Colon Rectum 2000; 43: 267-71.

Received: 27.06.2012, accepted: 27.12.2012. 